\title{
A UNIMODALITY RESULT IN THE ENUMERATION OF SUBGROUPS OF A FINITE ABELIAN GROUP
}

\author{
LYNNE M. BUTLER
}

(Communicated by Thomas H. Brylawski)

\begin{abstract}
The number of subgroups of order $p^{k}$ in an abelian group $G$ of order $p^{n}$ is a polynomial in $p, \alpha_{\lambda}(k ; p)$, determined by the type $\lambda$ of $G$. It is well known that $\alpha_{\lambda}(k ; p)=\alpha_{\lambda}(n-k ; p)$. Using a recent result from the theory of Hall-Littlewood symmetric functions, we prove that $\alpha_{\lambda}(k ; p)$, $0 \leq k \leq n$, is a unimodal sequence of polynomials. That is, for $1 \leq k \leq n / 2$, $\alpha_{\lambda}(k ; p)-\alpha_{\lambda}(k-1 ; p)$ is a polynomial in $p$ with nonnegative coefficients.
\end{abstract}

1. Finite abelian $p$-groups. If $G$ is an abelian group of order $p^{n}, p$ a prime, then $G$ is isomorphic to a direct product of cyclic groups

$$
\mathbf{Z} / p^{\lambda_{1}} \mathbf{Z} \times \mathbf{Z} / p^{\lambda_{2}} \mathbf{Z} \times \cdots \times \mathbf{Z} / p^{\lambda_{l}} \mathbf{Z} .
$$

The partition $\lambda=\left(\lambda_{1}, \lambda_{2}, \ldots, \lambda_{l}, 0, \ldots\right)$ where $\lambda_{1} \geq \lambda_{2} \geq \ldots \geq \lambda_{l}$ is called the type of $G$. We write $\lambda \vdash n$ since $\sum \lambda_{i}=n$.

If $H$ is a subgroup of $G$ and the type of $H$ is $\nu$, then $\nu_{i} \leq \lambda_{i}$ for all $i$. The number of subgroups of type $\nu$ is (using, e.g., $[2,8.1]$ )

$$
\prod_{i \geq 1} p^{\nu_{i+1}^{\prime}\left(\lambda_{i}^{\prime}-\nu_{i}^{\prime}\right)}\left[\begin{array}{c}
\lambda_{i}^{\prime}-\nu_{i+1}^{\prime} \\
\nu_{i}^{\prime}-\nu_{i+1}^{\prime}
\end{array}\right]_{p}
$$

where $\lambda_{i}^{\prime}=\operatorname{dim}_{\mathbf{Z} / p \mathbf{Z}}\left(p^{i-1} G / p^{i} G\right), \nu_{i}^{\prime}=\operatorname{dim}_{\mathbf{Z} / p \mathbf{Z}}\left(p^{i-1} H / p^{i} H\right)$ and $\left[\begin{array}{l}n \\ k\end{array}\right]_{p}$ is the number of $k$-dimensional subspaces of an $n$-dimensional vector space over $\mathbf{Z} / p \mathbf{Z}$. Combinatorially, $\lambda^{\prime}$ and $\nu^{\prime}$ are the conjugate partitions of $\lambda$ and $\nu$, respectively, and $\left[\begin{array}{l}n \\ k\end{array}\right]_{p}$ is the $p$-binomial coefficient (see, e.g., $[1$, p. 81 and p. 78]).

It follows immediately from (1) that the number of subgroups of order $p^{k}$ in a finite abelian $p$-group of type $\lambda, \alpha_{\lambda}(k ; p)$, is a polynomial in $p$ with nonnegative (integer) coefficients. It is well known (see, e.g., $\left[10\right.$, p. 87]) that $\alpha_{\lambda}(k ; p)=$ $\alpha_{\lambda}(n-k ; p)$. In fact, if $g_{\mu \nu}^{\lambda}(p)$ is the number of subgroups $H$ of type $\nu$ in a finite abelian $p$-group $G$ of type $\lambda$ such that $G / H$ is of type $\mu$, then P. Hall (see [10, p. 93]) showed:

(i) $g_{\mu \nu}^{\lambda}(p)$ is a polynomial in $p$ with integer coefficients;

(ii) $g_{\mu \nu}^{\lambda}(p)=g_{\nu \mu}^{\lambda}(p)$;

Received by the editors November 20, 1985 and, in revised form, September 3, 1986.

1980 Mathematics Subject Classification (1985 Revision). Primary 05A15, Secondary $20 \mathrm{~K} 01$.

Key words and phrases. Unimodal, Hall-Littlewood symmetric function, Kostka polynomial, symmetric chain order, Sperner property.

On leave for 1987-88 at the Institute for Mathematics and Its Applications, University of Minnesota, Minneapolis, Minnesota 55455. 
(iii) If $c_{\mu \nu}^{\lambda}$ is the Littlewood-Richardson coefficient [10, p. 68],

$$
\begin{aligned}
c_{\mu \nu}^{\lambda}=0 \Rightarrow & g_{\mu \nu}^{\lambda}(p)=0 ; \\
c_{\mu \nu}^{\lambda} \neq 0 \Rightarrow & g_{\mu \nu}^{\lambda}(p) \text { has degree } n(\lambda)-n(\mu)-n(\nu) \text { and leading } \\
& \text { coefficient } c_{\mu \nu}^{\lambda}, \text { where } n(\lambda)=\sum(i-1) \lambda_{i} .
\end{aligned}
$$

The aim of this paper is to prove the following result:

THEOREM. Let $\lambda \vdash n$ and $1 \leq k \leq n / 2$. Then $\alpha_{\lambda}(k ; p)-\alpha_{\lambda}(k-1 ; p)$ has nonnegative coefficients.

Thus, as conjectured by, e.g., P. Massell and M. Gikas, for fixed $\lambda \vdash n$ and $p$ the sequence $\alpha_{\lambda}(0 ; p), \alpha_{\lambda}(1 ; p), \ldots, \alpha_{\lambda}(n ; p)$ is unimodal.

2. Hall-Littlewood symmetric functions. P. Hall (see $[10$, p. 112]) indirectly defined symmetric functions $P_{\lambda}(x ; t), x=\left(x_{1}, x_{2}, \ldots\right)$, with the property that

$$
P_{\mu}(x ; t) P_{\nu}(x ; t)=\sum_{\lambda} g_{\mu \nu}^{\lambda}\left(t^{-1}\right) t^{n(\lambda)-n(\mu)-n(\nu)} P_{\lambda}(x ; t) .
$$

D. E. Littlewood [9] gave a direct definition of these symmetric functions, called Hall-Littlewood symmetric functions. Macdonald [10, Chapter 3] derives the following facts from Littlewood's expression. First, if $\Lambda$ is the ring of symmetric functions in $x=\left(x_{1}, x_{2}, \ldots\right)$ [10, p. 10], then $\left\{P_{\lambda}(x ; t)\right\}_{\lambda}$ is a $\mathbf{Z}[t]$-basis of $\Lambda \otimes \mathbf{Z}[t]$ $\left[10\right.$, p. 105]. Second, if $h_{n}(x)=\sum_{i_{1} \leq \cdots \leq i_{n}} x_{i_{1}} \cdots x_{i_{n}}$ is the homogeneous symmetric function, then

$$
h_{n}(x)=\sum_{\lambda \vdash n} t^{n(\lambda)} P_{\lambda}(x ; t)
$$

(see $[10$, p. 117]).

Finally, Macdonald [10, p. 129] states Lascoux and Schützenberger's result [8] that when the Schur function $s_{\rho}$ is expanded in terms of Hall-Littlewood symmetric functions

$$
s_{\rho}(x)=\sum_{\lambda} K_{\rho, \lambda}(t) P_{\lambda}(x ; t)
$$

the polynomials $K_{\rho, \lambda}(t)$ have nonnegative coefficients. In [5, Chapter 3] we discuss and complete Lascoux and Schützenberger's combinatorial proof [12].

3. Proof of the main theorem. We desire to show that if $\lambda \vdash n$ and $1 \leq$ $k \leq n / 2$, then $\alpha_{\lambda}(k ; p)-\alpha_{\lambda}(k-1 ; p)$ has nonnegative coefficients. It follows from (2) and (3) that the polynomial $\alpha_{\lambda}\left(k ; t^{-1}\right) t^{n(\lambda)}$ is the coefficient of $P_{\lambda}(x ; t)$ when the homogeneous symmetric function $h_{(n-k, k)}(x)$ is expanded in terms of the HallLittlewood symmetric functions. So,

$$
h_{(n-k, k)}(x)-h_{(n-k+1, k-1)}(x)=\sum_{\lambda \vdash n}\left(\alpha_{\lambda}\left(k ; t^{-1}\right)-\alpha_{\lambda}\left(k-1 ; t^{-1}\right)\right) t^{n(\lambda)} P_{\lambda}(x ; t) .
$$

On the other hand, the Jacobi-Trudi identity (see, e.g., [10, p. 25]) asserts that $s_{\rho}=\operatorname{det}\left|h_{\rho_{i}-i+j}\right|$. Hence

$$
h_{(n-k, k)}(x)-h_{(n-k+1, k-1)}(x)=s_{(n-k, k)}(x) .
$$


Since the $P_{\lambda}(x ; t)$ are linearly independent, comparing the two equations above yields

$$
\left(\alpha_{\lambda}\left(k ; t^{-1}\right)-\alpha_{\lambda}\left(k-1 ; t^{-1}\right)\right) t^{n(\lambda)}=K_{(n-k, k), \lambda}(t) .
$$

The result of Lascoux and Schützenberger now is seen to imply that $\alpha_{\lambda}(k ; p)-$ $\alpha_{\lambda}(k-1 ; p)$ has nonnegative coefficients.

NoTE. Using, e.g., [10, pp. 105, 130-131], we find that for $1 \leq k \leq n / 2$,

$$
\begin{array}{ll}
\alpha_{\lambda}(k ; p)=\alpha_{\lambda}(k-1 ; p) & \text { if } k>\sum_{i \geq 2} \lambda_{i}, \\
\alpha_{\lambda}(k ; p) \equiv \alpha_{\lambda}(k-1 ; p)+p^{k}\left(\bmod p^{k+1}\right) & \text { otherwise. }
\end{array}
$$

4. Chains of subgroups. Let $\lambda \vdash n$ and $S=\left\{a_{1}, a_{2}, \ldots, a_{j}\right\} \subseteq\{1,2, \ldots$, $n-1\}$ where $a_{1}<a_{2}<\cdots<a_{j}$. Define $\alpha_{\lambda}(S ; p)$ to be the number of chains of subgroups

$$
\{e\} \subset H_{1} \subset H_{2} \subset \cdots \subset H_{j} \subset G
$$

in a finite abelian $p$-group $G$ of type $\lambda$, where $H_{i}$ has order $p^{a_{i}}$. By $(1), \alpha_{\lambda}(S ; p)$ is a polynomial in $p$ with nonnegative coefficients. Define

$$
\beta_{\lambda}(S ; p)=\sum_{T \subseteq S}(-1)^{|S-T|} \alpha_{\lambda}(T ; p) .
$$

Since the lattice of subgroups of a finite abelian group is modular (see, e.g., [1, p. $42]$ ), it follows, e.g. from $[4,2.2]$, that for fixed $p$ we have $\beta_{\lambda}(S ; p) \geq 0$. We claim in fact that $\beta_{\lambda}(S ; p)$ has nonnegative coefficients as a polynomial in $p$. The following proof is due to R. Stanley and inspired the proof of the main theorem of this paper.

First notice that (2) and (3) yield

$$
h_{a_{1}}(x) h_{a_{2}-a_{1}}(x) \cdots h_{n-a_{j}}(x)=\sum_{\lambda \vdash n} \alpha_{\lambda}\left(S ; t^{-1}\right) t^{n(\lambda)} P_{\lambda}(x ; t) .
$$

Now consider the skew shape $\left[\mathbf{1 0}\right.$, p. 4] $\rho$ having $a_{1}$ squares in row one, $a_{2}-a_{1}$ squares in row two, $\ldots, n-a_{j}$ squares in row $j+1$, with consecutive rows overlapping by one square. (Thus $\rho$ is a border strip [10, p. 31].) Expand the skew Schur function $s_{\rho}[10$, p. 39] in terms of the homogeneous symmetric functions using the Jacobi-Trudi identity $[\mathbf{1 0}, \mathrm{p} .40]$. Then, using (4), rewrite $s_{\rho}$ as a linear combination of Hall-Littlewood symmetric functions to obtain

$$
s_{\rho}(x)=\sum_{\lambda \vdash n} \beta_{\lambda}\left(S ; t^{-1}\right) t^{n(\lambda)} P_{\lambda}(x ; t) .
$$

The result of Lascoux and Schützenberger, together with the fact that $s_{\rho}$ is a nonnegative linear combination of Schur functions (see, e.g., [10, p. 68]), now is seen to imply that $\beta_{\lambda}(S ; p)$ has nonnegative coefficients.

In [5, Chapter 2] we provide a simple combinatorial proof which uses only the expression given in (1) for the number of subgroups of type $\nu$ in a finite abelian $p$-group of type $\lambda$.

REMARK. Lascoux and Schützenberger define a statistic, charge, on tableaux $T$ of shape $\rho$ and weight $\lambda$ such that

$$
K_{\rho, \lambda}(t)=\sum_{T} t^{\operatorname{charge}(T)}
$$


The definition of charge as well as its relationship to Kostka polynomials $K_{\rho, \lambda}(t)$ extend naturally when $\rho$ is a skew shape. (See [5, Chapter 3].) Hence, injections $\psi_{\lambda, k}$ from border strip tableaux of weight $\lambda \vdash n \geq 2 k$ with $k-1$ squares in row one and $n-k+1$ squares in row two to border strip tableaux with $k$ squares in row one and $n-k$ squares in row two, such that charge $(\psi(T))=\operatorname{charge}(T)$, provide a combinatorial version of the proof of our main theorem. We describe such an injection $\psi$ : Add an entry $\infty$ at the end of row one of $T$. Slide row one of $T$ to the left above row two until some entry $y$ is immediately above an entry $x$ with $x \leq y$. Stop sliding; select the leftmost such pair of entries $x$ and $y$. The entries in row one of $\psi(T)$ are the entries in row one of $T$ together with the entry $x$ from row two of $T$.

This injection is inspired by Schützenberger's Jeu de Taquin (see, e.g. [5, Chapter 3]).

5. Open problems. An immediate consequence of our main result is that the lattice of subgroups (ordered by inclusion) of any finite abelian group is rankunimodal. (The product of two rank-unimodal, rank-symmetric, finite, graded posets is again rank-unimodal and rank-symmetric. See, e.g., [7].) The lattice of subgroups of a finite abelian $p$-group of type $\lambda=(1,1, \ldots, 1)$ is a symmetric chain order (see, e.g., $[1,8.63$, p. 433]. It has been conjectured that this result holds when $\lambda=(r, r, \ldots, r)$. A symmetric chain order is not only rank-unimodal and rank-symmetric, but also has property $\mathrm{S}$, a strong version of the Sperner property [7]. In general (e.g. for $\lambda=(2,1)$ ), the lattice of subgroups of a finite abelian $p$-group need not even have the Sperner property. For further information concerning the relationship between rank-unimodality and rank-symmetry, Sperner-type properties, and chain decomposition properties, see [7].

One can ask whether the lattice of subgroups of a finite abelian $p$-group is ranklog-concave. (This is easily seen to be true for type $\lambda=(1,1, \ldots, 1) \vdash n$ when $\alpha_{\lambda}(k ; p)$ is the $p$-binomial coefficient $\left[\begin{array}{l}n \\ k\end{array}\right]_{p}$.) In fact, calculations invite the stronger conjecture that

$$
\left(\alpha_{\lambda}(k ; p)\right)^{2}-\alpha_{\lambda}(k-1 ; p) \alpha_{\lambda}(k+1 ; p)
$$

has nonnegative coefficients as a polynomial in $p$, for all $\lambda \vdash n>k>0$. This conjecture is not even settled for $p$-binomial coefficients, but we have the weaker result that

$$
\left(\left[\begin{array}{l}
n \\
k
\end{array}\right]_{p}\right)^{2}-p\left[\begin{array}{c}
n \\
k-1
\end{array}\right]_{p}\left[\begin{array}{c}
n \\
k+1
\end{array}\right]_{p}
$$

has nonnegative coefficients. Our argument relies on a combinatorial expression, due to Carlitz [6], for the $p$-binomial coefficient

$$
\left[\begin{array}{l}
n \\
k
\end{array}\right]_{p}=\sum_{w \in S\left(1^{k} 2^{n-k}\right)} p^{\operatorname{inv}(w)}
$$

where $S\left(1^{k} 2^{n-k}\right)$ is the set of permutations of the multiset $\left\{1^{k}, 2^{n-k}\right\}$ and $\operatorname{inv}(w)$ is the inversion number of the multiset permutation $w=w_{1} w_{2} \cdots w_{n}$. We construct an injection

$$
\begin{array}{cccc}
\varphi: S\left(1^{k-1} 2^{n-k+1}\right) \times S\left(1^{k+1} 2^{n-k-1}\right) & \rightarrow & S\left(1^{k} 2^{n-k}\right) \times S\left(1^{k} 2^{n-k}\right) \\
(\pi, \sigma) & \mapsto & & \left(\pi_{L} \sigma_{R}, \sigma_{L} \pi_{R}\right)
\end{array}
$$


using an idea of Bhatt and Leiserson [3]. Just define

$$
\varphi(\pi, \sigma)=\left(\pi_{1} \cdots \pi_{i} \sigma_{i+1} \cdots \sigma_{n}, \sigma_{1} \cdots \sigma_{i} \pi_{i+1} \cdots \pi_{n}\right)
$$

where $i$ is smallest with $\pi_{1} \cdots \pi_{i} \sigma_{i+1} \cdots \sigma_{n} \in S\left(1^{k} 2^{n-k}\right)$. Observe that

$$
\operatorname{inv}\left(\pi_{L} \sigma_{R}\right)+\operatorname{inv}\left(\sigma_{L} \pi_{R}\right)=\operatorname{inv}(\pi)+\operatorname{inv}(\sigma)+1
$$

For a detailed proof and a straightforward generalization see [5]. Sagan [11] independently discovered the idea behind the injection $\varphi$ and gives injective proofs of log-concavity (in $k$ ) of the Stirling numbers, $c(n, k)$ and $S(n, k)$, as well as the binomial coefficients $\left(\begin{array}{l}n \\ k\end{array}\right)$. Our result in (5) is weaker than the conjecture that $\left(\left[\begin{array}{l}n \\ k\end{array}\right]_{p}\right)^{2}-\left[\begin{array}{c}n \\ k-1\end{array}\right]_{p}\left[\begin{array}{c}n \\ k+1\end{array}\right]_{p}$ has nonnegative coefficients; it is weaker because the sequence of coefficients in $\left[\begin{array}{l}n \\ l\end{array}\right]_{p}$ (hence in $\left(\left[\begin{array}{c}n \\ k\end{array}\right]_{p}\right)^{2}$ and in $\left[\begin{array}{c}n \\ k-1\end{array}\right]_{p}\left[\begin{array}{c}n \\ k+1\end{array}\right]_{p}$ ) is symmetric and unimodal, and the degree of $\left(\left[\begin{array}{c}n \\ k\end{array}\right]_{p}\right)^{2}$ exceeds the degree of $\left[\begin{array}{c}n \\ k-1\end{array}\right]_{p}\left[\begin{array}{c}n \\ k+1\end{array}\right]_{p}$ by 2 . In [13], Stanley gives an elegant proof that $\left[\begin{array}{l}n \\ k\end{array}\right]_{p}$ has unimodal coefficients; it is not known whether $\alpha_{\lambda}(k ; p)$ has unimodal coefficients for all $\lambda$ and $k$. See [5] for tables of these polynomials.

ACKNOWLEDGEMEnt. The author would like to thank Richard Stanley for his unrestrained contribution of ideas to the research discussed here.

\section{REFERENCES}

1. M. Aigner, Combinatorial theory, Springer-Verlag, New York, 1979.

2. Garrett Birkhoff, Subgroups of abelian groups, Proc. London Math. Soc. (2) 38 (1934-35), 385-401.

3. S. N. Bhatt and C. E. Leiserson, How to assemble tree machines, Proceedings of the 14th Symposium on Theory of Computing, San Francisco, May 5-7, 1982, pp. 77-84.

4. A. Björner, A. M. Garsia, and R. P. Stanley, An introduction to Cohen-Macaulay partially ordered sets, Ordered Sets, edited by I. Rival, Reidel, Dordrecht and Boston, Mass., 1982, pp. 583-615.

5. L. M. Butler, Combinatorial properties of partially ordered sets associated with partitions and finite abelian groups, Ph.D. thesis, M. I. T., 1986.

6. L. Carlitz, Sequences and inversions, Duke Math. J. 37 (1970), 193-198.

7. J. R. Griggs, On chains and Sperner $k$-families in ranked posets, J. Combinatorial Theory A28 (1980), 156-168.

8. A. Lascoux and M.-P. Schützenberger, Sur une conjecture de H. O. Foulkes, C. R. Acad. Sci. Paris 286A (1978), 323-324.

9. D. E. Littlewood, On certain symmetric functions, Proc. London Math. Soc. (3) 11 (1961), 485-498.

10. I. G. Macdonald, Symmetric functions and Hall polynomials, Oxford University Press, 1979.

11. B. E. Sagan, Inductive and injective proofs of log-concavity results, preprint.

12. M.-P. Schützenberger, Propriétés nouvelles des tableaux de Young, Séminaire Delange-PisotPoitou, $19^{e}, 1977 / 78$, no. 26, Secrétariat Mathématique, Paris.

13. R. Stanley, Some aspects of groups acting on finite posets, J. Combinatorial Theory $\mathbf{A 3 2}$ (1982), 132-161.

Department of Mathematics, Massachusetts Institute of Technology, CAMBRIDGE, MASSACHUSETTS 02139

Current address: Department of Mathematics, Fine Hall-Washington Road, Princeton University, Princeton, New Jersey 08544 